\title{
Checklist of the Cecidomyiidae (Diptera) of Finland
}

\author{
Mathias Jaschhof', Marcela Skuhravá2, Jouni Penttinen ${ }^{3}$
}

I Thälmann-Ring 64, D-17491 Greifswald, Germany 2 Bitovská 1227/9, 14000 Praha 4, Czech Republic

3 Metsähallitus, Natural Heritage, P.O. Box 36, FIN-40101 Jyväskylä, Finland

Corresponding author: Mathias Jaschhof (mjaschhof@yahoo.de)

Academic editor:J. Kahanpää | Received 13 March 2014 | Accepted 3 April 2014 | Published 19 September 2014

http://zoobank.org/DE94E3A3-65AD-4CB9-A6AB-33BB1980CDC3

Citation: Jaschhof M, Skuhravá M, Penttinen J (2014) Checklist of the Cecidomyiidae (Diptera) of Finland. In: Kahanpää J, Salmela J (Eds) Checklist of the Diptera of Finland. ZooKeys 441: 103-118. doi: 10.3897/zookeys.441.7503

\begin{abstract}
A list of the 356 species of Cecidomyiidae (Diptera) recorded from Finland is presented, which comprises 6 Lestremiinae, 156 Micromyinae, 16 Winnertziinae, 69 Porricondylinae, and 109 Cecidomyiinae. The faunistic knowledge of Finnish Winnertziinae, Porricondylinae and Cecidomyiinae is regarded as particularly poor. Based on species numbers known from other countries in Europe, a conservative estimate is 700-800 species of Cecidomyiidae actually occurring in Finland.
\end{abstract}

\section{Keywords}

Gall midges, Finland, species list

\section{Introduction}

With a world total of more than 6200 species described, Cecidomyiidae are among the largest families of Diptera, although adult cecidomyiids are mostly delicate midges with body lengths of less than $5 \mathrm{~mm}$. The family has been catalogued (Gagné and Jaschhof 2014) and that publication provides bibliographic and other information on all known species. Six subfamilies are recognised: the fungivorous Catotrichinae, Lestremiinae, Micromyinae, Winnertziinae and Porricondylinae, and the mostly herbivorous Cecidomyiinae. The latter subfamily, which also includes fungivores and predators, comprises the largest gall-making group of insects (hence the family name that translates as gall midges).

Copyright Mathias Jaschhof et al. This is an open access article distributed under the terms of the Creative Commons Attribution License (CC BY 4.0), which permits unrestricted use, distribution, and reproduction in any medium, provided the original author and source are credited. 
In the fungivorous subfamilies, many larvae are associated with dead wood, rendering those a biodiverse, albeit little studied group of saproxylic insects. In Cecidomyiinae, larvae associated with living flowering plants are usually restricted to development on one, or a few closely-related host species; predators are also generally assumed to be highly host-specific, as far as is known.

The Palaearctic has more named Cecidomyiidae species than any other zoogeographical region, and the best known gall midge fauna is that of Europe, with more than 1800 species (Skuhravá 2006). Even so, European Cecidomyiidae are far from being completely known, and the Finnish fauna provides a good example. To illustrate, while the previous checklist of Finnish Cecidomyiidae (Hackman 1980) comprised only four species of Lestremiinae and Micromyinae, a recent taxonomic study increased that number to 162, including 34 new species (Jaschhof and Jaschhof 2009). As for Winnertziinae and Porricondylinae, 27 species in Hackman's (1980) list compare with 69 species recorded here (Penttinen and Siitonen 2005, Penttinen and Spungis 2007, Penttinen and Jaschhof 2009; Jaschhof, unpublished; Penttinen, unpublished), yet much remains to be discovered, as is testified by specimens in the Penttinen Collection belonging to a number of unnamed species. In Cecidomyiinae, only three species (Hulden 2003; Skuhravá, unpublished) are here added to the Hackman list, a meager increase that is obviously explained by the slight research effort in recent years.

Identification of cecidomyiids, both larvae and adults, is generally difficult and usually left to a few taxonomic specialists of the family. An adult key to the Palaearctic genera of Cecidomyiidae was provided by Skuhravá (1997); to the Holarctic genera of Lestremiinae and Micromyinae by Jaschhof and Jaschhof (2009), and to the Holarctic genera of Winnertziinae and Porricondylinae by Jaschhof and Jaschhof (2013). Adult keys and illustrations by Jaschhof and Jaschhof $(2009,2013)$ can be used to identify the Finnish species of the fungivorous subfamilies. Adult keys to identify species of Cecidomyiinae in Finland are only available for a few small groups, so any attempts in this regard rely largely on descriptions and illustrations scattered in an extensive literature. In general, many Cecidomyiinae species cannot be identified unless a bundle of information (regarding host, gall, immature stages, adults of both sexes) are known. Illustrated keys to galls, including those caused by gall midges, have been published for Central and Northern Europe by Buhr (1964, 1965), a resource that is currently being revised and updated for publication in English in 2015 (see http:// www.pflanzengallen.de).

The arrangement of taxa in this list largely follows the recent edition of the world catalogue (Gagné and Jaschhof 2014) except that genera are here arranged under their pertinent tribes and supertribes, if known. All Finnish records of species of the fungivorous subfamilies were revised in recent years (Jaschhof and Jaschhof 2009, 2013; Penttinen, unpublished), so can be related to actual voucher specimens deposited in museum collections. The list of Cecidomyiinae, which is not updated to the same extent, is compiled from published locality records based on varying evidence, including galls alone. 


\section{Number of species:}

World: 6203 species (Gagné and Jaschhof 2014)

Europe: 1800 species (Skuhravá 2006)

Finland: 356 species (this paper)

Faunistic knowledge level in Finland: poor

\section{Checklist}

Nematocera Dumeril, 1805

infraorder Bibionomorpha Hennig, 1954

superfamily Sciaroidea Billberg, 1820

CECIDOMYIIDAE Newman, 1834

LESTREMIINAE Rondani, 1840

tribe Lestremiini Rondani, 1840

ALLARETE Pritchard, 1951

Allarete nigra Mamaev, 1994

ANARETE Haliday, 1833

Anarete sp. 2 [see Note below]

ANARETELLA Enderlein, 1911

Anaretella defecta (Winnertz, 1870)

= magnicornis Mamaev, 1964

Anaretella iola Pritchard, 1951

LESTREMIA Macquart, 1826

Lestremia cinerea Macquart, 1826

Lestremia leucophaea (Meigen, 1818)

MICROMYINAE Rondani, 1856

tribe Acoenoniini Pritchard, 1960

ACOENONIA Pritchard, 1947

Acoenonia europaea Mamaev, 1964

tribe Aprionini Jaschhof, 1998

APRIONUS Kieffer, 1894

Aprionus accipitris Jaschhof, 1997

Aprionus acutus Edwards, 1938

Aprionus adventitius Jaschhof, 2009

Aprionus aquilonius Jaschhof, 2009

Aprionus arcticus Mamaev, 2001

Aprionus aviarius Mamaev \& Berest, 1990

Aprionus betulae Jaschhof, 1996

Aprionus bidentatus (Kieffer, 1894)

Aprionus bifidus Mamaev, 1963

Aprionus bispinosus Edwards, 1938 
Aprionus brachypterus Edwards, 1938

Aprionus brevitegminis Jaschhof, 2009

Aprionus cardiophorus Mamaev, 1963

Aprionus carinatus Jaschhof, 1996

Aprionus complicatus Mamaev \& Berest, 1986

Aprionus confusus Mamaev, 1969

Aprionus corniculatus Mamaev, 1963

Aprionus cornutus Berest, 1986

Aprionus dalarnensis Mamaev, 1998

Aprionus dentifer Mamaev, 1965

Aprionus dispar Mamaev, 1963

Aprionus dissectus Mamaev \& Berest, 1990

Aprionus duplicatus Mamaev, 1998

Aprionus ensiferus Jaschhof, 1996

Aprionus fennicus Jaschhof, 2009

Aprionus flavidus (Winnertz, 1870)

Aprionus foliosus Jaschhof, 2009

Aprionus gladiator Jaschhof, 2009

Aprionus halteratus (Zetterstedt, 1852)

Aprionus heothinos Jaschhof, 2009

Aprionus hintelmannorum Jaschhof, 2009

Aprionus inquisitor Mamaev, 1963

Aprionus insignis Mamaev, 1963

Aprionus laevis Mohrig, 1967

Aprionus lapponicus Jaschhof \& Mamaev, 1997

Aprionus laricis Mamaev \& Jaschhof, 1997

Aprionus latitegminis Jaschhof, 2009

Aprionus longicollis Mamaev, 1963

Aprionus longitegminis Yukawa, 1967

Aprionus marginatus Mamaev, 1963

Aprionus miki Kieffer, 1895

Aprionus oligodactylus Jaschhof, 2009

Aprionus piceae Jaschhof, 1997

Aprionus praecipuus Jaschhof, 2009

Aprionus pyxidiifer Mamaev, 1998

Aprionus reduncus Jaschhof, 2009

Aprionus separatus Mamaev \& Jaschhof, 1997

Aprionus sievertorum Jaschhof, 2009

Aprionus similis Mamaev, 1963

Aprionus spiniferus Mamaev \& Berest, 1990

Aprionus spiniger (Kieffer, 1894)

Aprionus stiktos Jaschhof, 2009

Aprionus stylifer Mamaev, 1998 
Aprionus styloideus Mamaev \& Berest, 1990

Aprionus subacutus Jaschhof, 1997

Aprionus svecicus Jaschhof, 1996

Aprionus taigaensis Jaschhof, 2009

Aprionus tiliamcorticis Mamaev, 1963

Aprionus victoriae Jaschhof, 2009

tribe Bryomyiini Berest, 1993

BRYOMYIA Kieffer, 1895

= Tomonomyia Berest, 1993

Bryomyia apsectra Edwards, 1938

Bryomyia bergrothi Kieffer, 1895

Bryomyia gibbosa (Felt, 1907)

Bryomyia producta (Felt, 1908)

HETEROGENELLA Mamaev, 1963

= Cervuatina Berest, 1993

Heterogenella finitima Mamaev, 1998

Heterogenella hybrida Mamaev, 1963

Heterogenella linearis Yukawa, 1971

SKUHRAVIANA Mamaev, 1963

Skuhraviana triangulifera Mamaev, 1963

tribe Campylomyzini Kieffer, 1898

CAMPYLOMYZA Meigen, 1818

Campylomyza abbreviata Jaschhof, 2009

Campylomyza aemula Mamaev, 1998

Campylomyza alpina Siebke, 1863

Campylomyza arcuata Jaschhof, 2009

Campylomyza armata Mamaev, 1963

Campylomyza cavitata Mamaev, 1998

Campylomyza cingulata Jaschhof, 2009

Campylomyza dilatata Felt, 1907

Campylomyza falcifera Jaschhof, 2009

Campylomyza flavipes Meigen, 1818

= pallipes Zetterstedt, 1850

Campylomyza furva Edwards, 1938

Campylomyza fusca Winnertz, 1870

Campylomyza inornata Jaschhof, 2009

Campylomyza insolita Jaschhof, 2009

Campylomyza ormerodi (Kieffer, 1913)

Campylomyza serrata Jaschhof, 1998

Campylomyza spatulata Mamaev, 1998

Campylomyza stegetfore Jaschhof, 2009

EXCRESCENTIA Mamaev \& Berest, 1991

Excrescentia mutuata Mamaev \& Berest, 1991 
NEUROLYGA Rondani, 1840

= Cordylomyia Felt, 1911

Neurolyga acuminata Jaschhof, 2009

Neurolyga bilobata (Mamaev \& Rozhnova, 1982)

Neurolyga excavata (Yukawa, 1967)

Neurolyga interrupta Jaschhof, 2009

Neurolyga lonsdalensis Jaschhof, 2009

Neurolyga paludosa Jaschhof, 2009

Neurolyga sylvestris (Felt, 1907)

Neurolyga verna (Mamaev, 1963)

tribe Catochini Edwards, 1938

CATOCHA Haliday, 1833

Catocha incisa Jaschhof, 2009

Catocha latipes Haliday, 1833

tribe Micromyini Rondani, 1856

MICROMYA Rondani, 1840

Micromya lucorum Rondani, 1840

MONARDIA Kieffer, 1895

= Pezomyia Kieffer, 1913

sg. Monardia Kieffer, 1895

Monardia abnormis Mamaev, 1963

Monardia armata Jaschhof, 2003

Monardia lignivora (Felt, 1907)

Monardia obsoleta Edwards, 1938

Monardia pediculata (Mamaev, 1993)

Monardia stirpium Kieffer, 1895

Monardia yasumatsui Yukawa, 1967

sg. Trichopteromyia Williston, 1896

Monardia magnifica Mamaev, 1963

Monardia relicta Jaschhof, 2009

sg. Xylopriona Kieffer, 1913

Monardia adentis Jaschhof, 1998

Monardia atra (Meigen, 1804)

Monardia monotheca Edwards, 1938

Monardia radiella Mamaev, 1993

Monardia toxicodendri (Felt, 1907)

Monardia unguifera Berest \& Mamaev, 1997

POLYARDIS Pritchard, 1947

Polyardis adela Pritchard, 1947

Polyardis bispinosa (Mamaev, 1963)

Polyardis silvalis (Rondani, 1840)

tribe Peromyiini Kleesattel, 1979

PEROMYIA Kieffer, 1894 
Peromyia abdita Jaschhof, 2009

Peromyia abnormis Mamaev \& Berest, 1990

Peromyia albicornis (Meigen, 1830)

Peromyia anatina Mamaev \& Berest, 1990

Peromyia angellifera Jaschhof, 1997

Peromyia anisotoma Mamaev, 1994

Peromyia apposita Jaschhof, 1997

Peromyia bicolor (Edwards, 1938)

Peromyia bidentata Berest, 1998

Peromyia boreophila Jaschhof, 2001

Peromyia caricis (Kieffer, 1901)

Peromyia concitata Mamaev \& Berest, 1994

Peromyia cornuta (Edwards, 1938)

Peromyia curta Jaschhof, 1997

Peromyia diadema Mamaev, 1963

Peromyia edwardsi Berest, 1994

Peromyia fagiphila Jaschhof, 1997

Peromyia fungicola (Kieffer, 1901)

Peromyia intermedia (Kieffer, 1895)

Peromyia mitrata Jaschhof, 1997

Peromyia modesta (Felt, 1907)

Peromyia monilis Mamaev, 1965

Peromyia muscorum (Kieffer, 1895)

Peromyia ovalis (Edwards, 1938)

Peromyia palustris (Kieffer, 1895)

Peromyia perpusilla (Winnertz, 1870)

Peromyia photophila (Felt, 1907)

Peromyia pumila Jaschhof, 2001

Peromyia ramosa (Edwards, 1938)

Peromyia ramosoides Jaschhof, 2009

Peromyia sanguinea (Kieffer, 1894)

Peromyia scirrhosa Jaschhof, 2009

Peromyia scutellata Mamaev, 1990

Peromyia semotoides Jaschhof, 2009

Peromyia subbicolor Jaschhof, 2009

Peromyia subborealis Jaschhof, 1997

Peromyia syltefjordensis Jaschhof, 1996

Peromyia truncata Yukawa, 1967

Peromyia tundrae Jaschhof, 1996

Peromyia upupoides Jaschhof, 1997

WINNERTZIINAE Panelius, 1965

tribe Diallactiini Jaschhof, 2013

DIALLACTIA Gagné, 2004 
= Diallactes Kieffer, 1894 preocc.

Diallactia crocea (Kieffer, 1894)

= obscuripes (Spungis, 1985)

SYLVENOMYIA Mamaev \& Zaitzev, 1998

Sylvenomyia fennica Penttinen \& Jaschhof, 2009

Sylvenomyia spinigera (Spungis, 1985)

tribe Heteropezini Schiner, 1868

HETEROPEZA Winnertz, 1846

Heteropeza pygmaea Winnertz, 1846

LEPTOSYNA Kieffer, 1894

Leptosyna similis Jaschhof, 2013

MIASTOR Meinert, 1864

Miastor metraloas Meinert, 1864

tribe Winnertziini Panelius, 1965

KRONOMYIA Felt, 1911

Kronomyia ovalis (Mamaev, 1964)

RHIPIDOXYLOMYIA Mamaev, 1964

Rhipidoxylomyia brevicornis Mamaev, 1964

WINNERTZIA Rondani, 1860

Winnertzia bulbifera Mamaev, 1963

Winnertzia curvata Panelius, 1965

Winnertzia fusca Kieffer, 1901

Winnertzia globifera Mamaev, 1963

Winnertzia graduata Spungis, 1992

Winnertzia nigripennis Kieffer, 1896

Winnertzia rotundata Spungis, 1992

Winnertzia solidaginis Felt, 1907

= calciequina Felt, 1907

PORRICONDYLINAE Kieffer, 1913

tribe Asynaptini Rübsaamen \& Hedicke, 1926

ASYNAPTA Loew, 1850

Asynapta breviata Spungis, 1988

Asynapta inflata Spungis, 1988

Asynapta magdalini Panelius, 1965

Asynapta pectoralis (Winnertz, 1853)

Asynapta rufomaculata Panelius, 1965

Asynapta saliciperda Felt, 1908

= populnea Panelius, 1965

Asynapta strobi (Kieffer, 1920)

CAMPTOMYIA Kieffer, 1894

Camptomyia abnormis Mamaev, 1961

Camptomyia calcarata Mamaev, 1964

Camptomyia corticalis (Loew, 1851) 
Camptomyia flavocinerea Panelius, 1965

Camptomyia gigantea Spungis, 1989

Camptomyia pinicola Mamaev, 1961

Camptomyia piptopori Panelius, 1965

Camptomyia regia Spungis, 1989

Camptomyia salicicola Mamaev, 1961

= populicola Mamaev, 1961

Camptomyia spinifera Mamaev, 1961

COLOMYIA Kieffer, 1892

Colomyia clavata Kieffer, 1892

PARASYNAPTA Panelius, 1965

Parasynapta intermedia Panelius, 1965

tribe Dicerurini Mamaev, 1966

= Solntseviini Mamaev, 1966

DICERURA Kieffer, 1898

Dicerura furculata Mamaev, 1968

Dicerura mixta Spungis, 1987

Dicerura rossica (Mamaev, 1960)

Dicerura triangularis Mamaev, 1966

Dicerura unidentata Spungis, 1987

DIRHIZA Loew, 1850

Dirhiza lateritia Loew, 1850

HILVERSIDIA Mamaev, 1966

Hilversidia autumnalis Mamaev, 1966

PARATETRANEUROMYIA Spungis, 1987

Paratetraneuromyia vernalis Spungis, 1987

SOLNTSEVIA Mamaev, 1965

Solntsevia nigripes Mamaev, 1965

TETRANEUROMYIA Mamaev, 1964

Tetraneuromyia lenticularis (Spungis, 1987)

tribe Porricondylini Kieffer, 1913

= Holoneurini Enderlein, 1936

ARCTEPIDOSIS Mamaev, 1990

Arctepidosis paneliusi Mamaev \& Zaitzev, 1998

CASSIDOIDES Mamaev, 1960

Cassidoides fulvus (Kieffer, 1896)

= pini Mamaev, 1960

CLASPETTOMYIA Grover, 1964

= Pachylabis Panelius, 1965

Claspettomyia hamata (Felt, 1907)

Claspettomyia niveitarsis (Zetterstedt, 1850)

Claspettomyia rossica Mamaev, 1998

Claspettomyia ussuriensis Mamaev, 1998 
COCCOPSILIS Harris, 2004

$=$ Coccopsis Meijere preocc.

Coccopsilis marginata (Meijere, 1901)

Coccopsilis obscura (Mamaev, 1964)

Coccopsilis paneliusi (Yukawa, 1971)

DENDREPIDOSIS Mamaev, 1990

Dendrepidosis longipennis (Spungis, 1981)

DIVELLEPIDOSIS Fedotova \& Sidorenko, 2007

Divellepidosis armilla (Mamaev, 1994)

Divellepidosis fuscostriata (Panelius, 1965)

Divellepidosis hypoxantha (Panelius, 1965)

Divellepidosis lutescens (Spungis, 1981)

Divellepidosis pallescens (Panelius, 1965)

Divellepidosis taigacola Jaschhof, 2013

JAMALEPIDOSIS Mamaev, 1990

Jamalepidosis spungisi Jaschhof, 2013

MONEPIDOSIS Mamaev, 1966

Monepidosis furcata Mamaev, 1966

Monepidosis pectinata Mamaev, 1966

PANELIUSIA Jaschhof, 2013

Paneliusia albimanoides Jaschhof, 2013

Paneliusia aurantiaca (Panelius, 1965)

$=$ modesta (Spungis, 1981)

PAREPIDOSIS Kieffer, 1913

Parepidosis arcuata Mamaev, 1964

= longinodis Panelius, 1965

Parepidosis venustior Gagné, 2004

PAURODYLA Jaschhof, 2013

Paurodyla tyresta Jaschhof, 2013

PORRICONDYLA Rondani, 1840

Porricondyla fulvescens Panelius, 1965

Porricondyla neglecta Mamaev in Mamaev \& Krivosheina, 1965

Porricondyla nigripennis (Meigen, 1830)

Porricondyla rufescens Panelius, 1965

PSEUDEPIDOSIS Mamaev, 1966

Pseudepidosis trifida Mamaev, 1966

ROSTELLATYLA Jaschhof, 2013

Rostellatyla rostellata (Panelius, 1965)

ROSTRATYLA Jaschhof, 2013

Rostratyla globosa (Spungis, 1981)

SCHISTONEURUS Mamaev, 1964

Schistoneurus impressus Mamaev, 1964

Schistoneurus irregularis Mamaev, 1964 
SERRATYLA Jaschhof, 2013

Serratyla furcata (Mamaev, 2001)

Serratyla pubescens (Walker, 1856)

Serratyla spinosa Jaschhof, 2013

SPUNGISOMYIA Mamaev \& Zaitzev, 1996

Spungisomyia media (Spungis, 1981)

ZAITZEVIOLA Fedotova \& Sidorenko, 2007

Zaitzeviola latistylata Jaschhof, 2013

Zaitzeviola pilosistylata Jaschhof, 2013

Zaitzeviola rufocinerea (Panelius, 1965)

CECIDOMYIINAE Newman, 1834

supertribe Cecidomyiidi Rübsaamen \& Hedicke, 1925

tribe Aphidoletini Harris, 1966

APHIDOLETES Kieffer, 1904

Aphidoletes aphidimyza (Rondani, 1847)

Aphidoletes urticaria (Kieffer, 1895)

MONOBREMIA Kieffer, 1912

Monobremia subterranea (Kieffer, 1898)

tribe Asphondyliini Rübsaamen \& Hedicke, 1925

ASPHONDYLIA Loew, 1850

Asphondylia serpylli Kieffer, 1898

= thymi Kieffer, 1898

KIEFFERIA Mik, 1895

Kiefferia pericarpiicola (Bremi, 1847)

SCHIZOMYIA Kieffer, 1889

Schizomyia galiorum Kieffer, 1889

tribe Cecidomyiini Rübsaamen \& Hedicke, 1925

ANISOSTEPHUS Rübsaamen, 1917

Anisostephus betulinus (Kieffer, 1889)

CECIDOMYIA Meigen, 1803

Cecidomyia pini (De Geer, 1776)

CONTARINIA Rondani, 1860

Contarinia coryli (Kaltenbach, 1859)

Contarinia craccae Loew, 1850

Contarinia gei Kieffer, 1909

= geicola Rübsaamen, 1917

Contarinia kanervoi Barnes, 1958

Contarinia lonicerearum (F. Löw, 1877)

Contarinia loti (De Geer, 1776)

Contarinia merceri Barnes, 1930

Contarinia petioli (Kieffer, 1898)

Contarinia pisi (Loew, 1850)

Contarinia quercina (Rübsaamen, 1890) 
Contarinia sambuci (Kaltenbach, 1873)

= lonicerearum (F. Löw, 1874)

Contarinia tiliarum (Kieffer, 1890)

Contarinia tritici (Kirby, 1798)

Contarinia vincetoxici Kieffer, 1909

MACRODIPLOSIS Kieffer, 1895

Macrodiplosis pustularis (Bremi, 1847)

= dryobia $($ Löw, 1877)

Macrodiplosis roboris (Hardy, 1854)

= volvens Kieffer, 1895

PLEMELIELLA Seitner, 1908

Plemeliella abietina Seitner, 1908

STENODIPLOSIS Reuter, 1895

Stenodiplosis geniculati Reuter, 1895

THECODIPLOSIS Kieffer, 1895

Thecodiplosis brachyntera (Schwägrichen, 1835)

tribe Clinodiplosini Enderlein, 1936

AMETRODIPLOSIS Rübsaamen, 1910

Ametrodiplosis thalictricola (Rübsaamen, 1895)

CLINODIPLOSIS Kieffer, 1894

Clinodiplosis cilicrus (Kieffer, 1889)

SITODIPLOSIS Kieffer, 1913

Sitodiplosis mosellana (Géhin, 1857)

tribe Hormomyiini Rübsaamen \& Hedicke, 1925

PLANETELLA Westwood, 1840

Planetella ?grandis (Meigen, 1804)

tribe Lestodiplosini Harris, 1966

LESTODIPLOSIS Kieffer, 1894

Lestodiplosis pallidicornis Kieffer, 1898

unplaced Cecidomyiidi

HARMANDIOLA Skuhravá, 1997

= Harmandia Kieffer, 1896 preocc.

Harmandiola globuli (Rübsaamen, 1889)

Harmandiola tremulae (Winnertz, 1853)

= loewi (Rübsaamen, 1917)

HYGRODIPLOSIS Kieffer, 1912

Hygrodiplosis vaccinii (Kieffer, 1897)

MASSALONGIA Kieffer, 1897

Massalongia rubra (Kieffer, 1890)

RESSELIELLA Seitner, 1906

= Thomasiniana Strand, 1927

Resseliella ribis (Marikovskij, 1956)

Resseliella theobaldi (Barnes, 1927) 
XYLODIPLOSIS Kieffer, 1894

Xylodiplosis nigritarsis (Zetterstedt, 1850)

supertribe Lasiopteridi Rübsaamen \& Hedicke, 1925

tribe Dasineurini Rübsaamen \& Hedicke, 1925

DASINEURA Rondani, 1840

Dasineura affinis (Kieffer, 1886)

Dasineura alopecuri (Reuter, 1895)

Dasineura angelicae Rübsaamen, 1916

Dasineura cardaminis (Winnertz, 1853)

Dasineura engstfeldi (Rübsaamen, 1889)

Dasineura epilobii (F. Löw, 1889)

Dasineura fraxinea Kieffer, 1907

Dasineura fraxini (Bremi, 1847)

Dasineura galiicola (F. Löw, 1880)

Dasineura gentneri Pritchard, 1953

Dasineura hygrophila (Mik, 1883)

Dasineura hyperici (Bremi, 1847)

Dasineura jaapi Rübsaamen, 1914

Dasineura kiefferiana (Rübsaamen, 1891)

Dasineura leguminicola (Lintner, 1879)

Dasineura lotharingiae (Kieffer, 1888)

Dasineura mali (Kieffer, 1904)

Dasineura napi (Loew, 1850) = brassicae (Winnertz, 1853)

Dasineura populeti (Rübsaamen, 1889)

Dasineura pteridicola (Kieffer, 1901)

Dasineura pteridis (Muller, 1871) = filicina (Kieffer, 1889)

Dasineura pustulans (Rübsaamen, 1889)

Dasineura pyri (Bouché, 1847)

Dasineura ribis Barnes, 1940

Dasineura rosae (Bremi, 1847) $=$ Wachtliella rosarum (Hardy, 1854)

Dasineura similis (F. Löw, 1888)

Dasineura sisymbrii (Schrank, 1803)

Dasineura tetensi (Rübsaamen, 1892)

Dasineura thomasiana (Kieffer, 1888)

Dasineura tiliae (Schrank, 1803) = tiliamvolvens (Rübsaamen, 1889)

Dasineura trifolii (F. Löw, 1874)

Dasineura ulmaria (Bremi, 1847)

Dasineura urticae (Perris, 1840)

Dasineura viciae (Kieffer, 1888) 
Dasineura violae (F. Löw, 1880)

GEOCRYPTA Kieffer, 1913

Geocrypta galii (Loew, 1850)

GEPHYRAULUS Rübsaamen, 1915

Gephyraulus raphanistri (Kieffer, 1886)

GIRAUDIELLA Rübsaamen, 1915

Giraudiella inclusa (Frauenfeld, 1862)

JAAPIELLA Rübsaamen, 1915

Jaapiella loticola (Rübsaamen, 1889)

Jaapiella veronicae (Vallot, 1827)

KALTENBACHIOLA Hedicke, 1938

Kaltenbachiola strobi (Winnertz, 1853)

MACROLABIS Kieffer, 1892

Macrolabis cirsii (Rübsaamen, 1890)

Macrolabis orobi (F. Löw, 1877)

RABDOPHAGA Westwood, 1847

Rabdophaga dubiosa Kieffer, 1913

Rabdophaga gemmicola (Kieffer, 1896)

Rabdophaga heterobia (Loew, 1850)

Rabdophaga iteobia (Kieffer, 1890)

Rabdophaga marginemtorquens (Bremi, 1847)

Rabdophaga nervorum (Kieffer, 1895)

Rabdophaga rosaria (Loew, 1850)

Rabdophaga salicis (Schrank, 1803)

Rabdophaga terminalis (Loew, 1850)

WACHTLIELLA Rübsaamen, 1915

Wachtliella caricis (Loew, 1850)

= riparia (Winnertz, 1853)

= muricatae (Meade, 1886)

Wachtliella persicariae (Linnaeus, 1767)

tribe Lasiopterini Rübsaamen \& Hedicke, 1925

LASIOPTERA Meigen, 1818

Lasioptera populnea Wachtl, 1883

Lasioptera rubi (Schrank, 1803)

tribe Oligotrophini Rübsaamen \& Hedicke, 1925

OLIGOTROPHUS Latreille, 1805

Oligotrophus juniperinus (Linnaeus, 1758)

Oligotrophus panteli Kieffer, 1898

tribe Poomyini Rübsaamen \& Hedicke, 1925

MAYETIOLA Kieffer, 1896

Mayetiola avenae (Marchal, 1895)

Mayetiola destructor (Say, 1817)

Mayetiola joannisi Kieffer, 1896

tribe Rhopalomyiini Harris, 1966 
RHOPALOMYIA Rübsaamen, 1892

Rhopalomyia chrysanthemi (Ahlberg, 1939)

Rhopalomyia millefolii (Loew, 1850)

Rhopalomyia ptarmicae (Vallot, 1849)

Rhopalomyia tanaceticola (Karsch, 1879)

Rhopalomyia tubifex (Bouché, 1847)

unplaced Lasiopteridi

CYSTIPHORA Kieffer, 1892

Cystiphora sanguinea (Bremi, 1847)

= hieracii (F. Löw, 1874)

= pilosellae Kieffer, 1892

Cystiphora sonchi (Vallot, 1827)

ITEOMYIA Kieffer, 1913

Iteomyia capreae (Winnertz, 1853)

SEMUDOBIA Kieffer, 1913

Semudobia betulae (Winnertz, 1853)

\section{Excluded species}

Anarete lacteipennis Kieffer, 1906. Misidentification.

Camptomyia antennata Felt, 1920. Misidentification.

Camptomyia maxima Mamaev, 1961. Presumptive misidentification; Finnish record cannot be verified.

Camptomyia piceae Panelius, 1965. Presumptive misidentification; Finnish record cannot be verified.

Catarete brevinervis (Zetterstedt, 1851). Misidentification.

Cecidomyia aurora Mannerheim, 1823. Nomen dubium.

Epidosis flavescens F. Löw, 1874. Nomen dubium.

\section{Note}

Anarete sp. 2. This species was described by Jaschhof and Jaschhof (2009) but not named. Identification of Anarete species is practically impossible using the keys and descriptions available in the literature.

\section{Acknowledgements}

The subfamilies Lestremiinae and Micromyinae were edited by M.J., Winnertziinae and Porricondylinae by M.J. and J.P., and Cecidomyiinae by M.S. The manuscript was read and commented on by Keith M. Harris, Ripley, UK, and by two reviewers unknown to us. 


\section{References}

Buhr H (1964) Bestimmungstabellen der Gallen (Zoo- und Phytocecidien) an Pflanzen Mittel- und Nordeuropas. Band I. VEB Gustav Fischer Verlag, Jena, xv + 761 pp.

Buhr H (1965) Bestimmungstabellen der Gallen (Zoo- und Phytocecidien) an Pflanzen Mittel- und Nordeuropas. Band II. VEB Gustav Fischer Verlag, Jena, 763-1572. [25 pls. with unnumberered facing pages bearing figure legends]

Gagné RJ, Jaschhof M (2014) A Catalog of the Cecidomyiidae (Diptera) of the World. 3rd Edition. Digital version 2. http://www.ars.usda.gov/SP2UserFiles/Place/12454900/Gagne_2014_World_Cecidomyiidae_Catalog_3rd_Edition.pdf

Hackman W (1980) A check list of the Finnish Diptera. Notulae entomologicae 60: 17-48.

Hulden L (2003) Dasineura pustulans (Rübs.) (Cecidomyiidae) discovered in Finland. Sahlbergia 8(2): 100 .

Jaschhof M, Jaschhof C (2009) The Wood Midges (Diptera: Cecidomyiidae: Lestremiinae) of Fennoscandia and Denmark. Studia dipterologica Supplement 18. Ampyx-Verlag, Halle (Saale), 333 pp.

Jaschhof M, Jaschhof C (2013) The Porricondylinae (Diptera: Cecidomyiidae) of Sweden, with notes on extralimital species. Studia dipterologica Supplement 20. Ampyx-Verlag, Halle (Saale), 392 pp.

Penttinen J, Jaschhof M (2009) On the systematics of Sylvenomyia Mamaev \& Zaitzev (Diptera, Cecidomyiidae, "Porricondylinae"), with the description of a new species from Finland. Zootaxa 2032: 48-54.

Penttinen J, Siitonen J (2005) Five species of log midges (Diptera, Cecidomyiidae: Porricondylinae) new to Finland. Sahlbergia 10(2): 50-53.

Penttinen J, Spungis V (2007) Additions to the Finnish fauna of log midges (Diptera, Cecidomyiidae: Porricondylinae). Sahlbergia 12(1): 36-42.

Skuhravá M (1997) Family Cecidomyiidae. In: Papp L, Darvas B (Eds) Contributions to a Manual of Palaearctic Diptera (with Special Reference to Flies of Economic Importance). Volume 2: Nematocera and Lower Brachycera. Science Herald, Budapest, 71-204.

Skuhravá M (2006) Species richness of gall midges (Diptera: Cecidomyiidae) in the main biogeographical regions of the world. Acta Societatis Zoologicae Bohemicae 69: 327-372. 